\title{
Structural analysis of Ni-doped $\mathrm{SrTiO}_{3}$ : XRD study
}

\author{
Zdeněk Jansa, Lucie Prušáková, Fatima Alarab, Pavol Šutta, Ján Minár \\ ${ }^{1}$ New Technologies Research Centre, University of West Bohemia, Pilsen, Czech Republic \\ ${ }^{a)}$ Corresponding author: zjansa@ntc.zcu.cz \\ b)jminar@ntc.zcu.cz
}

\begin{abstract}
The aim of this work is to study the structure of Ni-doped $\mathrm{SrTiO}_{3}$ thin films by X-ray diffraction (XRD). All samples were prepared by magnetron sputtering on $\mathrm{Si}$ and $\mathrm{SiO} 2$ substrates. The main objective of this work is to monitor the crystallization of the deposited thin layer of Ni-doped $\mathrm{SrTiO}_{3}$. The X-ray diffraction measurements were done on the films as deposited and after annealing in vacuum up to $900^{\circ} \mathrm{C}$. The $\mathrm{x}$-ray analysis was used with both geometries (symmetric and asymmetric). Those measurements allow us to get information about the influence of $\mathrm{Ni}$ on the final structure, the size of crystallites, the micro-strains and the deformation of the lattice. In particular, here we domonstarate that Ni doping lead to the unique stabilisation of crystall growth of $\mathrm{SrTiO} 3$ as compared to the undoped $\mathrm{SrTiO}_{3}$.
\end{abstract}

\section{INTRODUCTION}

A Perovskite structure is calcium titanium oxide type of mineral, whose crystal structure is $\mathrm{ABX}_{3}$. Many different cations can be embedded in this structure, allowing the development of diverse engineered materials. In the structure the atoms $\mathrm{A}$ and $\mathrm{B}$ are two cations with very different sizes. The $\mathrm{X}$ is an anion that makes bonds with both cations [1].

One famous example is the paraelectric [2] oxide $\mathrm{SrTiO}_{3}$ which has been extensively studied during the past two decades because of its important and interesting properties for both technological applications and solid state physics. It has been known to have interesting and useful dielectric properties for almost 40 years [3][4]. Stoichiometric $\mathrm{SrTiO}_{3}$ (STO) is an insulator with a large band gap (about 3,2 eV at room temperature). However, the conductivity and photocatalytic capabilities of STO can be improved. The addition of dopants like rare earth ions, transition metal ions or other impurities make STO as a promising multifunctional material for various applications, in particularly, the ferromagnetic properties can be obtained by the incorporation of transition metal impurities such as $\mathrm{Fe}, \mathrm{Ni}, \mathrm{Co}$, etc. into the hosting material [7][8][9].

Only few insufficient theoretical studies are available to understand the Ni-doping effect on the electronic structures, optical properties and the possible origin of the high photocatalytic activity under visible light in $\mathrm{SrTiO}_{3}$. Experimentally the ferromagnetic response was obtained in Ni and Co-doped STO polycrystalline materials even at room temperature. Doping effect was seen on the structure when the crystallite size increased. The impurity atoms caused a shift of the diffraction lines. The doping effect had an influence on magneto-optical response with unsaturated hysteresis loop at an applied magnetic field. [10].

Future technological applications of STO are going hand in hand with the preparation methods to produce atomically flat STO (100) and (111) surface. Even-though, major milestones in growing epitaxial STO has been achieved [5], still it results to the step-and-terrace morphology of the surface [6]. 
In spite, $\mathrm{SrTiO}_{3}$ shows very interesting and unique physical and electronic structure properties as for example presence of 2DEG [4], until now detailed angle resolved photoemission studies of electronic structure over wide binding energy window for this system are missing, due to the above mentioned quality and crystalline of the surface.

The present work investigates the influence of incorporated $\mathrm{Ni}$ as a dopant of $\mathrm{SrTiO}_{3}$ thin films, studies the structural phases, the crystallite size, the micro-strain and the deformation of the lattice. To characterize the structure of the $\mathrm{SrTiO}_{3}$ thin films X-Ray diffraction was used. Here we show, that Ni dopant has stabilizing effect of the crystallinity and growth of STO films which will allow further future studies of electronic structure by surface sensitive techniques as for example angle resolved photoemission.

\section{EXPERIMENTAL METHODS}

All the STO films were prepared by the RF (13.56 MHz) magnetron sputtering using BOC Edwards TF 600 coating system. Prior the sputtering, the deposition chamber was evacuated to a base pressure of $2 \times 10^{-4} \mathrm{~Pa}$. Substrates were cleaned by ion etching in argon plasma by applying RF power of $200 \mathrm{~W}$ for 15 minutes at $0.2 \mathrm{~Pa}^{-\mathrm{SrTiO}_{3}} \operatorname{target}$ of 99,9\% purity as placed on the magnetron connected to RF power. Ni pellets were placed on the sputtering target surface. Different number of pellets have been used to achieve films with rising amount of Ni incorporated to the film structure. The deposition was kept under constant discharge RF power $(400 \mathrm{~W})$. The films were deposited on crystalline $\mathrm{Si}$ wafer and $\mathrm{SiO}_{2}$ substrate. The deposition was kept under argon atmosphere at constant pressure of 0.6 $\mathrm{Pa}$ for 60 minutes. Thickness of the film was in the range of 310 to $365 \mathrm{~nm}$.

The structure of the films was observed by X-ray diffraction (XRD) using an automatic powder diffractometer Panalytical X'Pert Pro with $\mathrm{Cu} \mathrm{K} \alpha$ X-ray tube $(\lambda=1,540598 \mathrm{~nm})$. XRD patterns were collected using two geometries. The first one is asymmetric geometry $\omega-2 \vartheta$, with $\omega=0,5^{\circ}$. The second one is symmetric geometry $\vartheta-\vartheta$. Both of symmetries were used in the range of 20 to 65 degrees for Si wafer and in the range of 15 to 75 degrees for $\mathrm{SiO}_{2}$ substrate, respectively. The difference between these geometries is, that in the case of asymmetric geometry we can see the diffraction of all planes fulfilling the Bragg's law $(\lambda=2 d \sin \theta)$ for actual 29 angle since in case of symmetric geometry only the planes parallel with the surface and satisfy the Bragg's law. In the first case the patterns were collected by point Xe detector and in the second case the high resolution ultra-fast semiconductor detector PIXcel was used.

A line profile analysis of the diffraction lines was performed in order to calculate the crystallite sizes, the microstrain and the deformation of the lattice. For these analyses the procedure based on a Voigt function was used. In order to obtain the line profile parameters, the line alignment with a Pearson VII was used.

The XRD measurements were done on the as deposited films and consequently after annealing. The films were annealed under vacuum $10^{-5} \mathrm{~Pa}$ at temperature of $900^{\circ} \mathrm{C}$. The temperature was increased by the steps of $10^{\circ} \mathrm{C}$ every 5 minutes up to $900{ }^{\circ} \mathrm{C}$. When the temperature reached $900{ }^{\circ} \mathrm{C}$, the 30 minutes holding time at the temperature was set. During this time the crystallization of former amorphous structure of thin films proceeded. The cooling of annealed samples was continuous from $900^{\circ} \mathrm{C}$ to room temperature.

\section{RESULTS AND DISCUSSION}

The XRD patterns were measured on five samples since there were five different contents of $\mathrm{Ni}$ in the $\mathrm{SrTiO}_{3}$ films. The first group of samples was on the silicon substrate and second group was on the $\mathrm{SiO}_{2}$ substrate. The samples were labeled with their atomic percent of $\mathrm{Ni}$ in the next text. The Ni content in here studied samples was $0.81,1.06$, $1.41,2.37$ and $4.43 \mathrm{wt} \%$ and has been experimentally proofed by corresponding EDS and XPS studies.

All the films under were first analyzed at as-deposited state by the asymmetric geometry. Figure 2. a) shows the

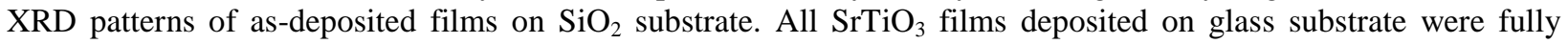
amorphous. Figure 1. b) shows XRD patterns of as-deposited films on Si wafer. These XRD patterns are different from previous ones. There are a sharp diffraction lines visible which become from substrate - so-called Laue peaks. Also some diffraction lines from $\mathrm{SrTiO}_{3}$ can be seen. These indicated an initial crystallization of the $\mathrm{SrTiO}_{3}$ phase. We can assume that the rising amount of $\mathrm{Ni}$ in the films initiated the crystallization process at low temperature. The influence of $\mathrm{Ni}$ on initial crystallization must be proven by the following analyzes. A standard of pure $\mathrm{SrTiO}_{3}$ was inserted into the second diffraction pattern. 


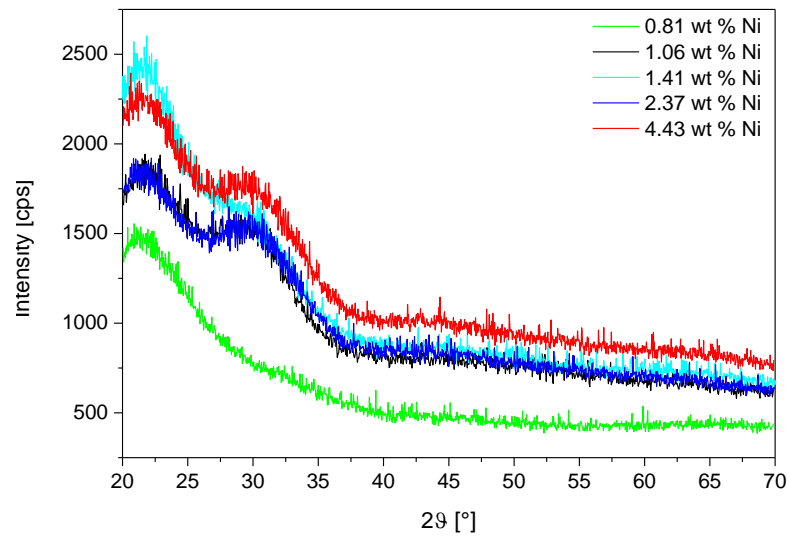

a)

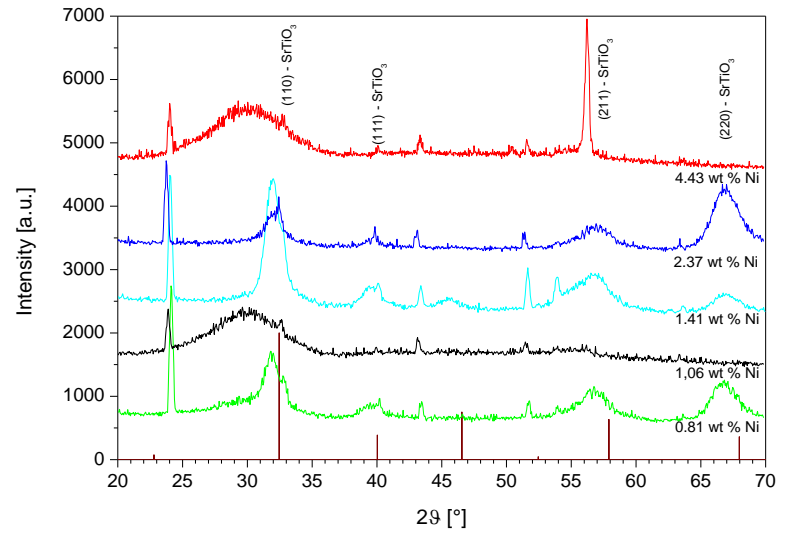

b)

FIGURE 1. Diffraction patterns of as-deposited $\mathrm{SrTiO}_{3}$ films a) on $\mathrm{SiO}_{2}$ substrate, b) on $\mathrm{Si}$ wafer

The second measurement was done after annealing and crystallization was performed during the annealing procedure.

The final result for films deposited on $\mathrm{SiO}_{2}$ substrate will be discussed first. XRD patterns are shown in Figure 2 a). There is a clear change at first sight with comparison with the XRD patterns in Figure 1 a). It was obviously growth of $2500 \mathrm{cps}$ up to $11000 \mathrm{cps}$ (compare Figure 1 and 4) due to crystallinity of samples. The diffraction lines of majority phase are clearly and easy readable. Other diffraction lines are from minority phase like a $\mathrm{Ni}\left(\mathrm{TiO}_{3}\right)$ or $\mathrm{SrO}$. There is a rest of amorphous phase from substrate at low angles of XRD patterns. The diffraction patterns of sample $0,81 \mathrm{wt} \%$ of $\mathrm{Ni}$ is different from others. There is a small intensity due to the smallest contain of $\mathrm{Ni}$, which is only $1.52 \mathrm{wt} \%$. In Figure 1b), the problem didn't occur at the same sample. The reason is, that the second sample is on the Si wafer, probably. The difference between XRD patterns in Figure 2 a) and b) is that the Si wafer samples don't contain the amorphous phase. In Figure 2 b) there are some XRD patterns called "Laue peaks". These patterns are from the single-crystal silicon substrate created by a residual continuous X-ray radiation and don't have an influence on thin films analyzed.

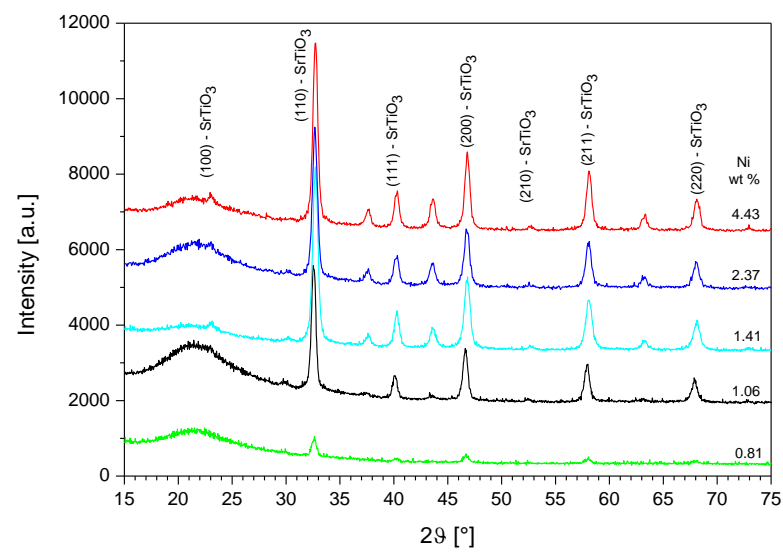

a)

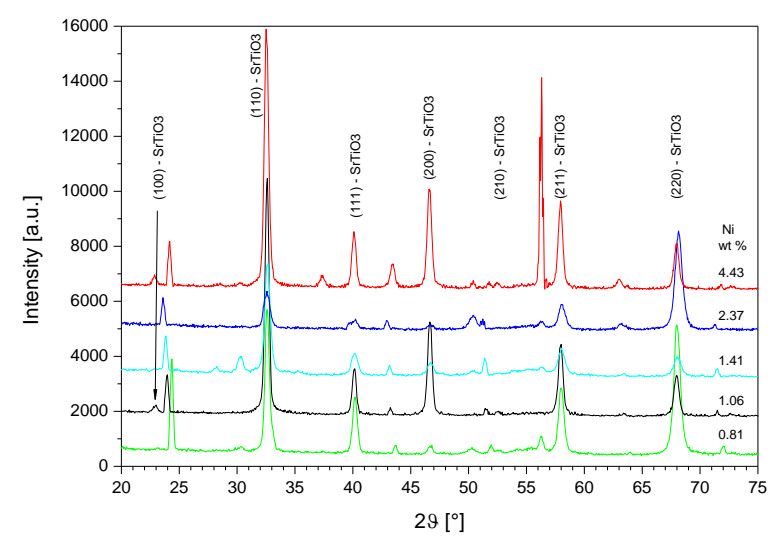

b)

FIGURE 2. Diffraction patterns of annealed $\mathrm{SrTiO}_{3}$ films a) on $\mathrm{SiO}_{2}$ substrate, b) on $\mathrm{Si}$ wafer

The evolution of grain size with Ni content is shown in Figure 3 a). The highest grain size was obtained Ni content $1 \mathrm{wt} \%$. For Ni content $2.37 \mathrm{wt} \%$ the diffraction line with highest integral intensity was (220). That is why it is considered in Figure 3 a). 
There is $\mathrm{SrTiO}_{3}$ as a majority phase visible in diffraction patterns of samples investigated. According to the high intensity of diffraction lines and narrow FWHM (full width at half maximum) there is a preferred orientation (texture) in [110] direction the domain size at the preferred orientation is higher than other direction.

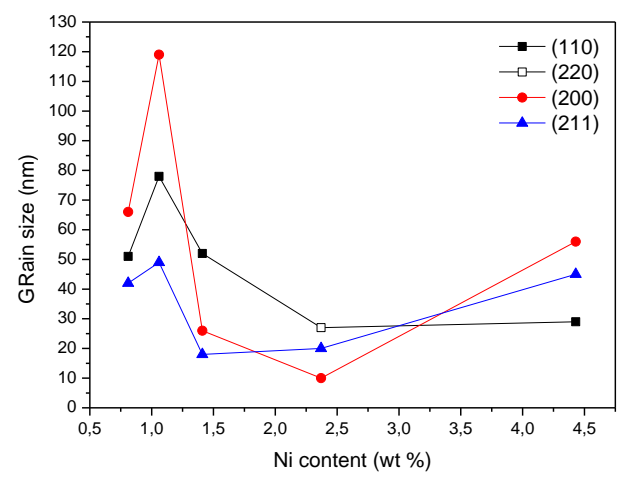

a)

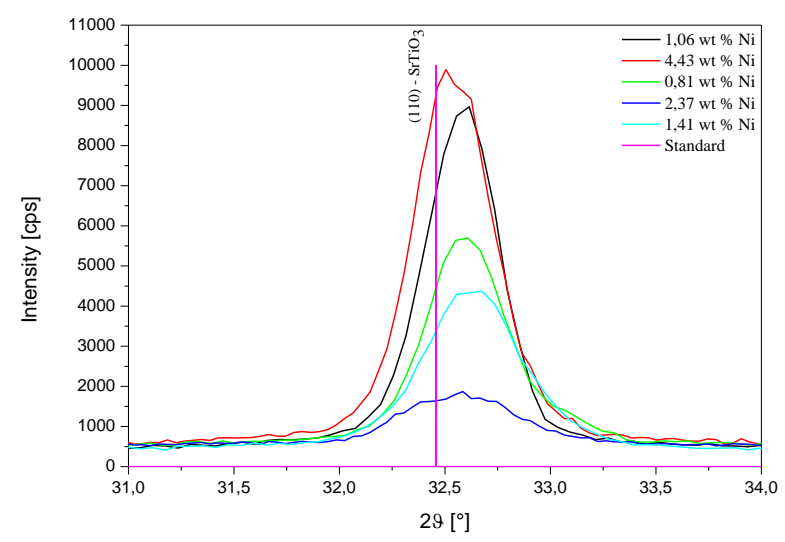

b)

FIGURE 3. a) Grain size evaluated from three diffraction lines as a function of Ni content in the thin films on Si wafer, b) diffraction lines (110) comparison with standard of pure $\mathrm{SrTiO}_{3}$

Figure $3 \mathrm{~b}$ ) shows displacement of diffraction line (110) ( in direction [110]) compared to standard. Diffraction lines for all of the samples are shifted to the right from the standard. It can be due to Ni-doped. The dopant atoms replace $\mathrm{Sr}$ atoms in the base lattice of $\mathrm{SrTiO}_{3}$ we assume [Chyba! Nenalezen zdroj odkazů.]. This causes tensile stress and shifts the diffraction line to the right. The displacement is evident in all diffraction lines of $\mathrm{SrTiO}_{3}$ which is visible in Figure 3 a). It can be caused by the crystallographic parameters, which are for $\mathrm{SrTiO}_{3}$ thin films with cubic lattice Pm-3m, a $=0,3898 \mathrm{~nm}$ and $\alpha=90^{\circ}$. The crystallographic parameters of Ni dopant are cubic lattice Fm-3m, $\mathrm{a}=$ 0,354 and $\alpha=90^{\circ}$. The atomic radius for $\mathrm{Sr}$ is $219 \mathrm{pm}$ and $\mathrm{Ni}$ is $184 \mathrm{pm}$, respectively.

The phase analysis of the sample 4,43 wt \% of Ni on Si wafer is in Table 1. Based on data in the table it is evident, that there are several phases in sample. In this case, there is the phase of $\mathrm{NiO}$ due to the higher content of $\mathrm{Ni}$ in sample. This phase didn't appear in other samples. Also a trace amount of $\mathrm{SrO}$ is present in this sample. Furthermore, crystallite sizes are apparent (Domain size). The size of crystallites corresponds with intensity of diffraction lines and grow with their value. The experimental intensity of the phases, and their comparison with standard can be seen in Table 1.

\begin{tabular}{|c|c|c|c|c|c|c|c|c|c|c|}
\hline \multirow{2}{*}{ Sample } & \multirow{2}{*}{ Gonimeter } & \multicolumn{2}{|c|}{ Position 29 [degrees] } & \multicolumn{3}{|c|}{ Intensity [\%] } & \multirow{2}{*}{$\begin{array}{c}\text { Domain size } \\
\langle\mathrm{D}\rangle[\mathrm{nm}]\end{array}$} & \multirow{2}{*}{\begin{tabular}{|c||} 
Micro-strains \\
$\langle\varepsilon>[-]$ \\
\end{tabular}} & \multirow{2}{*}{ Phase } & \multirow{2}{*}{ hkl } \\
\hline & & Standart & Experiment & Standart & Experiment & Relative exp. & & & & \\
\hline \multirow{14}{*}{$\begin{array}{c}4.43 \mathrm{wt} \% \\
\text { of } \mathrm{Ni}\end{array}$} & \multirow{14}{*}{$\omega-2 \vartheta$} & 22,795 & 22,875 & 3,5 & 1,52 & 3,8 & 29 & "0,0042 & 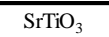 & 100 \\
\hline & & 32,457 & 32,539 & 100 & 40 & 100 & 64 & 0,0029 & $\mathrm{SrTiO}_{3}$ & 110 \\
\hline & & 40,031 & 40,101 & 19,3 & 8,45 & 21,13 & 64 & 0,0021 & $\mathrm{SrTiO}_{3}$ & 111 \\
\hline & & 46,56 & 46,619 & 37,5 & 16,32 & 40,8 & 56 & 0,0017 & $\mathrm{SrTiO}_{3}$ & 200 \\
\hline & & 52,448 & 52,478 & 2,2 & 0,93 & 2,33 & & & $\mathrm{SrTiO}_{3}$ & 210 \\
\hline & & 57,901 & 57,94 & 31,7 & 14,63 & 36,58 & 45 & 0,0009 & $\mathrm{SrTiO}_{3}$ & 211 \\
\hline & & 67,965 & 67,972 & 18 & 8,46 & 21,15 & 40 & 0,0003 & $\mathrm{SrTiO}_{3}$ & 220 \\
\hline & & \multicolumn{9}{|c|}{ Minority phases } \\
\hline & & \multicolumn{2}{|c|}{ Position 29 [degrees] } & \multicolumn{3}{|c|}{ Intensity [\%] } & \multirow{2}{*}{$\begin{array}{c}\text { Domain size } \\
\langle\mathrm{D}\rangle[\mathrm{nm}] \\
\end{array}$} & \multirow{2}{*}{\begin{tabular}{|c|} 
Micro-strains \\
$<\varepsilon>[-]$ \\
\end{tabular}} & \multirow{2}{*}{ Phase } & \multirow{2}{*}{ hkl } \\
\hline & & Standart & Experiment & Standart & Experiment & Relative & & & & \\
\hline & & 37,339 & 37,36 & 65 & 2,25 & 55,69 & & & $\mathrm{Ni}_{4.00} \mathrm{O}_{4.00}$ & 111 \\
\hline & & 43,385 & 43,432 & 100 & 4,04 & 100 & & & $\mathrm{Ni}_{4.00} \mathrm{O}_{4.00}$ & 002 \\
\hline & & 63,031 & 63,013 & 51,3 & 2,28 & 56,44 & & & $\mathrm{C} \mathrm{Ni}_{4.00} \mathrm{O}_{4.00}$ & 022 \\
\hline & & 50,538 & 50,397 & & 1,09 & & & & $\mathrm{Sr}_{4.00} \mathrm{O}_{4.00}$ & 111 \\
\hline
\end{tabular}

TABLE 1. Analysis of the sample 4,43 wt $\%$ of Ni on Si wafer

Empty cells in Table 1 at minority phases and others are due to the small content in the samples. The content is below the detection limit of the procedure used. 
The results diffraction analysis of all samples using the symmetrical geometry $(\vartheta-\vartheta)$ are analogical to previous. There is an insignificant difference in content of minority phases only. These results won't be presented for this reason.

\section{COCLUSION}

This work shows the diametric difference between the state of as-deposited samples of Ni-doped $\mathrm{SrTiO}_{3}$ thin films and the state after annealing. The XRD patterns for as-deposited samples on the $\mathrm{SiO} 2$ substrate show only amorphous phase. The films deposited on Si wafer are amorphous too with the presence of some polycrystalline phase of $\mathrm{SrTiO}_{3}$ at initial state. It is caused by Ni-doped in connection with $\mathrm{Si}$ wafer. It depends on the content on $\mathrm{Ni}$ in the structure.

The polycrystalline structure of Ni-doped $\mathrm{SrTiO}_{3}$ appeared after annealing under vacuum up to $900^{\circ} \mathrm{C}$. Nickel around 1 wt. \% leads to increasing of grain size. $\mathrm{SrTiO}_{3}$ containing more than $1.41 \mathrm{wt} \%$ Ni contain also few minority phases like as $\mathrm{SrO}, \mathrm{Ni}\left(\mathrm{TiO}_{3}\right), \mathrm{TiO}_{2}$ and $\mathrm{NiO}$. Grain size in these films is significantly lower.

Incorporation of $\mathrm{Ni}$ into the base lattice of $\mathrm{SrTiO}_{3}$ result in tensile stress in the structure. The diffraction lines are shifted to the right compared to the standard of pure $\mathrm{SrTiO}_{3}$ due to the tensile stress. The influence of the stress on the structure must be investigated, as well the influence of $\mathrm{Ni}$ content on the structure and other properties.

\section{ACKNOWLEDGMENTS}

This work was supported by CEDAMNF project (CZ.02.1.01/0.0/0.0/15_003/0000358), co-funded by the ERDF as part of the OP RDE program of the Ministry of Education, Youth and Sports (Czech Republic)

\section{REFERENCES}

1 Wenk, Hans-Rudolf; Bulakh, Andrei; Minerals: Their Constitution and Origin. New York, NY, Cambridge University Press. ISBN 978-0-521-52958-7, (2004).

2 Hong-Cheng L, Weidong Si, Rui-Lan Wang, Yi Xuan, Bao-Ting Liu, X.X Xi; Dielectric properties of $\mathrm{SrTiO}_{3}$ thin films grown on various perovskite electrodes by pulsed laser deposition in Materials Science and Engineering: B, Volume 56, Issues 2-3, 6 November 1998, Pages 218-222.

3 A. Anspoks et al.; Journal of Physics: Conference Series 712, 012101 (2016).

4 Sing et al., Eur. Phys. J. Special Topics 226, 2457-2475 (2017).

5 M. Kawasaki, K. Takahashi, T. Maeda, R. Tsuchiya, M. Shinohara, O. Ishiyama, T. Yonezawa, M. Yoshimoto, H. Koinuma; Atomic control of the SrTiO3 crystal surface, Science 266, 1540-1542 (1994).

6 Seyoung Cook, Kendra Letchworth-Weaver, I-Cheng Tung, Tassie K. Andersen, Hawoong Hong, Laurence D. Marks, Dillon D. Fong, Sci. Adv. 5, eaav0764 (2019).

7 S.Suzuki, T. Yamamoto, H. Suzuki, K. Kawaguchi, K Takahashi, Y. Yosisatho; Fabrication and characterization of Ba1-xKxBiO3/Nb doped SrTiO3 all-oxide-type Schotky junctions, Journal Applied Physics 81(1997).

8 M.P. Singh, W. Prellier, L. Mechin, C. Simon, B. Raveau; Correlation between structure and properties in multiferroic La0,7Ca0,3MnO3/BaTiO3 superlattice, Journal Applied Physics 99 (2) (2006) 024105.

9 S. Banerjee, A. Datta, A. Bhaumik, D. Chakravorty; Multiferroic behavior of nanoporous BaTiO3, Jornal Applied Physics, 110 (6) (2011) 064316.

10 R. Siddheswaran, Pavol Šutta, Petr Novák, Marie Netrvalová, Aleš Hendrych, Ondřej Životský; In-situ X-ray diffraction studies and magneto-opto Kerr effect on RF sputtered thin films of BaTiO3 na Co, Nb co-doped BaTiO3, in Ceramics International 42 (2016) 3882-3887.

11 M. Rizwan, A. Ali, Z.Usman, N.R. Khalid, H.B. Jin, C.B. CAO, Structural, electronic and optical properties of copper-doped SrTiO3 perovskite: A DFT study, Physica B: Condesed Matter, vol. 552, january 2019, page 5257. 